\title{
Flow over Exponentially Stretching Sheet through Porous Medium with Heat Source/Sink
}

\author{
I. Swain, ${ }^{1}$ S. R. Mishra, ${ }^{2}$ and H. B. Pattanayak ${ }^{3}$ \\ ${ }^{1}$ Department of Mathematics, KES, Bhubaneswar 751002, India \\ ${ }^{2}$ Department of Mathematics, I.T.E.R., Siksha 'O' Anusandhan University, Bhubaneswar, Odisha 751030, India \\ ${ }^{3}$ Department of Mathematics, Ravenshaw University, Bhubaneswar 753002, India
}

Correspondence should be addressed to S. R. Mishra; satyaranjan_mshr@yahoo.co.in

Received 2 September 2015; Accepted 4 November 2015

Academic Editor: Oronzio Manca

Copyright (C) 2015 I. Swain et al. This is an open access article distributed under the Creative Commons Attribution License, which permits unrestricted use, distribution, and reproduction in any medium, provided the original work is properly cited.

\begin{abstract}
An attempt has been made to study the heat and mass transfer effect in a boundary layer MHD flow of an electrically conducting viscous fluid subject to transverse magnetic field on an exponentially stretching sheet through porous medium. The effect of thermal radiation and heat source/sink has also been discussed in this paper. The governing nonlinear partial differential equations are transformed into a system of coupled nonlinear ordinary differential equations and then solved numerically using a fourth-order Runge-Kutta method with a shooting technique. Graphical results are displayed for nondimensional velocity, temperature, and concentration profiles while numerical values of the skin friction local Nusselt number and Sherwood number are presented in tabular form for various values of parameters controlling the flow system.
\end{abstract}

\section{Introduction}

The magnetohydrodynamics (MHD) heat and mass transfer from different geometry embedded in a porous medium are of interest for engineering and geographical applications such as geothermal reservoirs, thermal insulation, cooling of nuclear reactors, and enhanced oil recovery. Many chemical engineering processes like metallurgical and polymer extrusion processes involve cooling of molten liquid being stretched into a cooling system; the fluid mechanical properties of the penultimate product depend mainly upon the cooling liquid used and the rate of stretching. Some polymer fluids like polyethylene oxide and polyisobutylene solution in cetane, having better electromagnetic properties, are normally used as cooling liquid as their flow can be regulated by external magnetic fields in order to improve the quality of final product. Sakiadis $[1,2]$ investigated the boundary layer flow induced by a moving plate in a quiescent ambient fluid. Thereafter, various aspects of the problem have been investigated by many authors such as Fang [3], Fang and Lee [4], and White [5].
Buoyancy is also of importance in an environment where differences between heat and air temperatures can give rise to complicated flow patterns [6]. Furthermore, magnetohydrodynamic (MHD) has attracted the attention of a large number of scholars due to its diverse applications. Chamkha and Abdul-Rahim Khaled [7] have investigated the effects of magnetic field on natural convection flow past a vertical surface. Makinde [8] and Makinde et al. [9] have studied mass diffusion effects on natural convection flow past a flat plate. A comprehensive account of the boundary layers flow over a vertical plate embedded in a porous medium can be found in Kim and Vafai [10] and Liao and Pop [11].

It is well known that fluids such as water, mineral oil, and ethylene glycol for conventional heat transfer are poor conductors of heat compared to most solids. An innovative way of improving the heat transfer in fluids by suspending small solid particles in the fluids was introduced by Choi [12]. This new kind of fluids is named nanofluids which is a suspension of solid nanoparticles of diameter 1-100 nm in conventional heat transfer basic fluids such as water, oil, or ethylene glycol. It is believed that these fluids increase 
the heat transfer performance of the base fluid enormously. This characteristic feature of nanofluids is to enhance the thermal conductivity which is more useful to meet today's cooling rate requirements. A comprehensive survey of convective transport was presented by Buongiorno [13] by pointing out various facts concerning nanofluids. Similarity solution to heat and mass transfer analysis on MHD 3D water-based nanofluid was investigated by Baag and Mishra [14]. The study of magnetohydrodynamic (MHD) flow has many important industrial, technological, and geothermal applications such as high temperature plasmas, cooling of nuclear reactors, MHD accelerators and power generation systems, and liquid metal fluids. Magnetic nanofluids have colloidal suspensions containing magnetizable nanoparticles which have both the fluid and magnetic properties as well as thermal properties. Vajravelu and Rollins [15] analyzed heat transfer in an electrically conducting fluid over a stretching surface taking into account the magnetic field. Tripathy et al. [16] studied chemical reaction effect on MHD free convective surface over a moving vertical plane through porous medium. Mishra et al. [17] investigated the flow of heat and mass transfer on MHD free convection in a micropolar fluid with heat source.

Sparrow and Abraham [18] have investigated a new buoyancy model replacing the standard pseudo density difference for internal natural convection in gases. Sparrow and Abraham [19] used the relative velocity model where only one of the participating media is in motion. The steady laminar flow and heat transfer characteristics of a continuously moving vertical sheet of extruded material are studied close to and far downstream from the extrusion slot by Al-Sanea [20]. Soundalgekar and Ramana Murty [21] have discussed the effects of power law surface temperature variation on the heat transfer from a continuous moving surface with constant surface velocity. More recently, Cortell [22] extended the work of Afzal et al. [23] by taking viscous dissipation effect in the energy balance. The effects of transpiration on the flow and heat transfer over a moving permeable surface in a parallel stream are analyzed by Ishak et al. [24]. The development of the boundary layer on a fixed or moving surface parallel to a uniform free stream in presence of surface heat flux has been investigated by Ishak et al. [25]. Patil et al. [26] have examined the role of internal heat generation or absorption effects on the flow and heat transfer over a moving vertical plate. In this study, authors have considered the steady flow and heat transfer characteristics. Unsteady mixed convection flows do not necessarily possess similarity solutions in many practical applications. The unsteadiness and nonsimilarity in such flows may be due to the free stream velocity or due to the curvature of the body or due to the surface mass transfer or even possibly due to all these effects. Because of the mathematical difficulties involved in obtaining nonsimilar solutions for such problems, many investigators have confined their studies to either steady nonsimilar flows or unsteady semisimilar or self-similar flows.

In the present study we proposed to investigate the effect of heat source/sink on the free convection flow of a viscous incompressible electrically conducting fluid on a vertical plate with variable wall temperature and concentration.

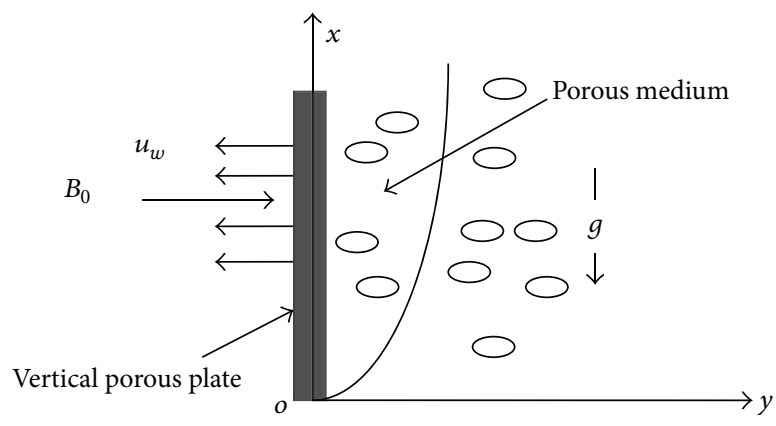

FIGURE 1: Flow geometry.

The effect of pertinent parameters is presented in both graphical and tabular form. It is noticed that the results obtained will not only provide useful information for applications, but also serve as a complement to Mabood et al. [27].

\section{Mathematical Formulation}

Consider a steady, laminar, incompressible, two-dimensional free convective heat and mass transfer along a semi-infinite vertical plate embedded in a doubly stratified, electrically conducting micropolar fluid. Choose the coordinate system such that the $x$-axis is along the vertical plate and the $y$ axis normal to the plate. The physical model and coordinate system are shown in Figure 1. The plate is maintained at temperature $T_{w}(x)$ and concentration $C_{w}(x)$. The temperature and the mass concentration of the ambient medium are assumed to be linearly stratified in the forms $T_{\infty}(x)=T_{\infty, 0}+$ $A_{1} x$ and $C_{\infty}(x)=C_{\infty, 0}+B_{1} x$, respectively, where $A_{1}$ and $B_{1}$ are constants and varied to alter the intensity of stratification in the medium and $T_{\infty, 0}$ and $C_{\infty, 0}$ are the beginning ambient temperature and concentration at $x=0$, respectively. A uniform magnetic field of magnitude $B_{0}$ is applied normal to the plate. The magnetic Reynolds number is assumed to be small so that the induced magnetic field can be neglected in comparison with the applied magnetic field.

Following Sparrow and Abraham [18] the Boussinesq and boundary layer approximations, the governing equations for the micropolar fluid are given by

$$
\begin{gathered}
\frac{\partial u}{\partial x}+\frac{\partial v}{\partial y}=0 \\
u \frac{\partial u}{\partial x}+v \frac{\partial u}{\partial y}=v \frac{\partial^{2} u}{\partial y^{2}}-\frac{\sigma B_{0}^{2}}{\rho}-\frac{v}{K_{p}^{\prime}} u, \\
u \frac{\partial T}{\partial x}+v \frac{\partial T}{\partial y}=\alpha \frac{\partial^{2} T}{\partial y^{2}}-\frac{1}{\rho c_{p}} \frac{\partial q_{r}}{\partial y}+\frac{Q}{\rho c_{p}}\left(T-T_{\infty}\right), \\
u \frac{\partial C}{\partial x}+v \frac{\partial C}{\partial y}=D \frac{\partial^{2} C}{\partial y^{2}},
\end{gathered}
$$

where $u$ and $v$ are the components of velocity along the $x$ and $y$ directions, respectively, $T$ is the temperature, $C$ is the concentration, $B_{0}$ is the coefficient of the magnetic field, $\mu$ is the dynamic coefficient of viscosity of the fluid, $K_{p}^{\prime}$ is 
the permeability of the fluid, $v$ is the kinematic viscosity, $\alpha$ is the thermal diffusivity, $Q$ is dimensional heat source, $D$ is the molecular diffusivity, $q_{r}$ is the radiative heat flux, and $c_{p}$ is the specific heat. have

By the use of Rosseland approximation for radiation, we

$$
\begin{gathered}
q_{r}=\frac{4 \sigma}{3 K^{*}} \frac{\partial T^{4}}{\partial y}, \\
T^{4} \approx 4 T_{\infty}^{3} T-3 T_{\infty}^{4} ;
\end{gathered}
$$

that is,

$$
q_{r}=\frac{4 \sigma}{3 K^{*}} \frac{\partial\left(4 T_{\infty}^{3} T-3 T_{\infty}^{4}\right)}{\partial y}=\frac{16 \sigma T_{\infty}^{3}}{3 K^{*}} \frac{\partial T}{\partial y} .
$$

Hence, (3) reduces to

$$
u \frac{\partial T}{\partial x}+v \frac{\partial T}{\partial y}=\left(\alpha+\frac{16 \sigma T_{\infty}^{3}}{3 K^{*} \rho C_{p}}\right) \frac{\partial^{2} T}{\partial y^{2}}+\frac{Q}{\rho C_{p}}\left(T-T_{\infty}\right)
$$

The boundary conditions are

$$
\begin{gathered}
u=U_{w}(x), \\
v=0, \\
T=T_{w}, \\
C=C_{w}, \\
\text { at } y=0 \\
u \longrightarrow 0, \\
T \longrightarrow T_{\infty}, \\
C \longrightarrow C_{\infty}, \\
\text { as } y \longrightarrow \infty,
\end{gathered}
$$

where the subscripts $w$ and $\infty$ indicate the conditions at wall and at the outer edge of the boundary layer, respectively.

\section{Method of Solution}

Continuity equation (1) is satisfied by introducing the stream function $\psi$ such that

$$
\begin{gathered}
u=\frac{\partial \psi}{\partial y} \\
v=-\frac{\partial \psi}{\partial x}
\end{gathered}
$$

In order to explore the possibility for the existence of similarity, we assume

$$
\begin{gathered}
\eta=y \sqrt{\frac{u_{0}}{2 \vartheta L}} e^{x / 2 L}, \\
u=u_{0} e^{x / L} f^{\prime}(\eta), \\
v=-\sqrt{\frac{\vartheta u_{0}}{2 L}} e^{x / 2 L}\left\{f(\eta)+\eta f^{\prime}(\eta)\right\}, \\
T=T_{\infty}+T_{0} e^{x / 2 L} \theta(\eta), \\
C=C_{\infty}+C_{0} e^{x / 2 L} \phi(\eta), \\
B=B_{0} e^{x / 2 L} .
\end{gathered}
$$

Substituting (9) and (10) in (2)-(7), it is found that similarity exists and hence we obtain

$$
\begin{gathered}
f^{\prime \prime \prime}+f f^{\prime \prime}-2 f^{\prime 2}-\left(M+\frac{1}{K_{p}}\right) f^{\prime}=0, \\
\left(1+\frac{4}{3} R\right) \theta^{\prime \prime}+\operatorname{Pr}\left(f \theta^{\prime}-f^{\prime} \theta+S \theta\right)=0, \\
\phi^{\prime \prime}+\operatorname{Sc}\left(f \phi^{\prime}-f^{\prime} \phi\right)=0,
\end{gathered}
$$

where primes denote differentiation with respect to similarity variable $\eta, \operatorname{Pr}=\nu / \alpha$ is the Prandtl number, $\mathrm{Sc}=\nu / D$ is the Schmidt number, $M=\sigma B_{0}^{2} / \mu B^{2}$ is the magnetic field parameter, and $1 / K_{p}=2 L \vartheta / u_{0} C_{p}$ is the porosity parameter. $R=4 \sigma T_{\infty}^{3} / K^{*} K$ is the radiation parameter and $S=Q / 2 L u \rho C_{p}$ is the source parameter.

Boundary conditions (8) in terms of $f, g, \theta$, and $\phi$ become

$$
\begin{gathered}
f(0)=0, \\
f^{\prime}(0)=1, \\
f^{\prime}(\infty)=0, \\
\theta(0)=1, \\
\phi(0)=1 .
\end{gathered}
$$

Physical Quantities of Interest. Local skin friction coefficient $C_{f}$ is defined as

$$
\frac{1}{\sqrt{2}} C_{f} \sqrt{R_{e}}=f^{\prime \prime \prime}(0)
$$


The heat and mass transfers from the plate, respectively, are given by

$$
\begin{gathered}
q_{w}=-k\left(\frac{\partial T}{\partial y}\right)_{y=0}, \\
q_{m}=-D\left(\frac{\partial C}{\partial y}\right)_{y=0}, \\
\frac{N_{u}}{\sqrt{R_{e}}}=-\theta^{\prime}(0), \\
\frac{S_{h}}{\sqrt{R_{e}}}=-\phi^{\prime}(0),
\end{gathered}
$$

where

$$
R_{e}=\frac{U_{w}}{\vartheta}
$$

\section{Results and Discussion}

The present study considers the MHD flow of a viscous incompressible electrically conducting fluid flow past an exponentially stretching sheet through a porous medium in the presence of transverse magnetic field and thermal radiation in the presence of uniform heat source/sink. The mass transfer analysis has also been discussed in this paper. The aim of the following discussion is to bring out the effect of permeability of the medium, plate temperature, and thermal radiation on the flow phenomena.

The heat generation/absorption contribute significantly for nonisothermal heat transfer case. Another consideration of the present study is the saturated porous media. Porous media are very widely used to insulate a heated body to maintain its temperature. They are considered to be useful in diminishing the natural free convection which would otherwise occur intensely on the vertical surface.

Figures 2 and 3 exhibit the variation of magnetic parameter and porous matrix on velocity and temperature profile. It is observed from Figure 2 that an increase in magnetic parameter reduces the velocity profile at all points in both the absence/presence of porous matrix. The present result is in good agreement with the result of Mabood et al. [27] for $K_{p}=100$ (dotted). However, from Figure 3, reverse effect is encountered in case of temperature distribution; that is, increases in magnetic parameter enhance the temperature profile at all points in both the absence and presence of porous matrix. An increase in temperature due to the presence of magnetic elements may be attributed to the fact that when fluid is in flow, a certain amount of energy is stored up in the material as strain energy in addition to thermal radiation but the reduction of velocity in the presence of magnetic field due to resistive Lorentz force which comes into play.

The effect of Prandtl number on temperature profile in the absence of source/sink is well marked in Figure 4. It is seen that an increase in Pr leads to decrease of the temperature in the absence of porous matrix $\left(K_{p}=100\right.$, dotted) whereas

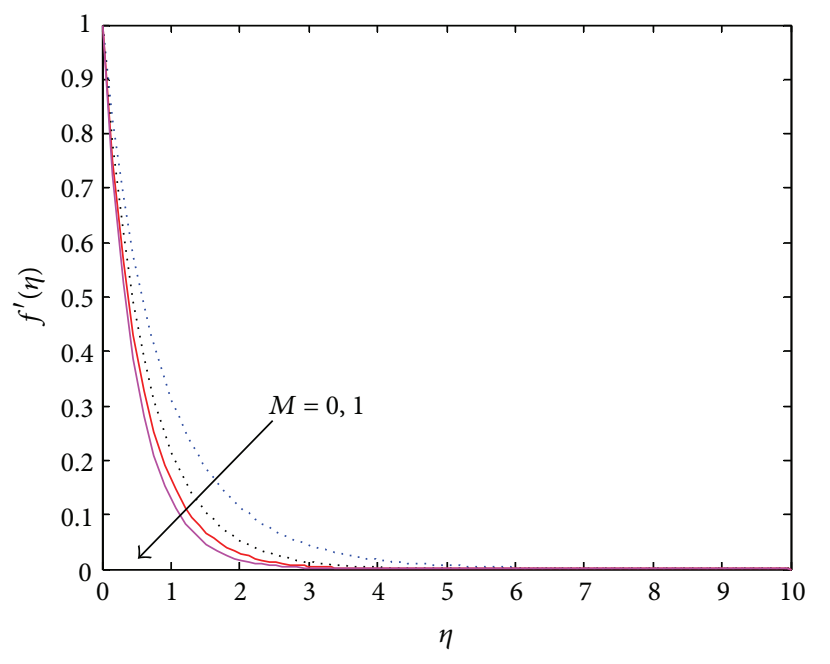

$\begin{aligned} \cdots \cdots & K_{p}=100 \\ -K_{p} & =0.5\end{aligned}$

Figure 2: Velocity distribution for different values of $M$.

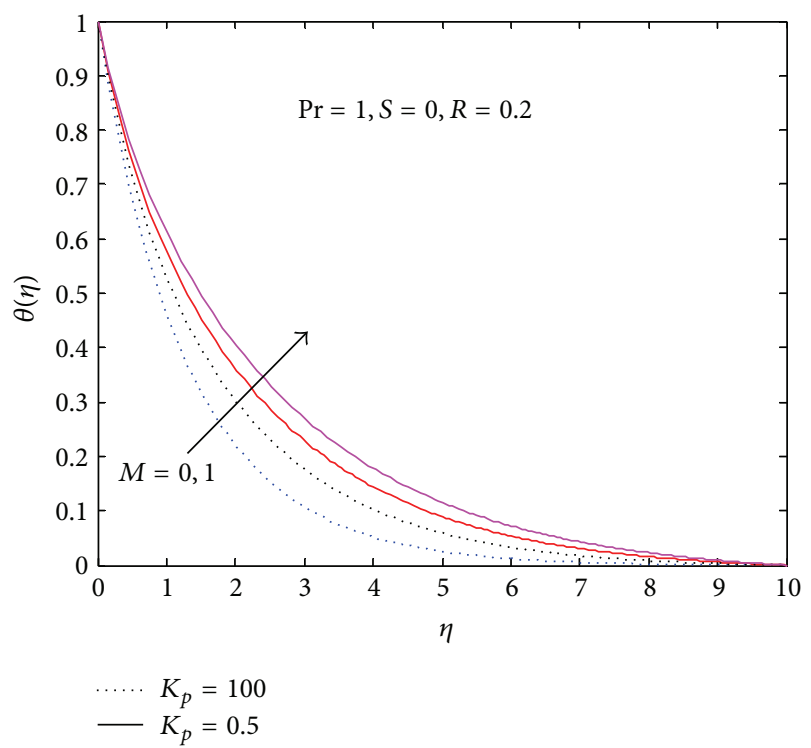

FIGURE 3: Temperature distribution for different values of $M$ and $K_{p}$.

the profile has its maximum value in the presence of porous matrix $\left(K_{p}=0.5\right.$, bold $)$.

Thus, it may be considered that the increase in Pr means slow rate of thermal diffusion. Thus, it may be concluded that thinning of thermal boundary layer thickness is the consequence of fluid with slow rate of thermal diffusion in the presence of magnetic field in the absence of porous matrix but the presence enhances it.

Figure 5 exhibits the effects of $R$ on the temperature field in the presence of heat source. It is clear from Figure 5 that in the absence of $K_{p}\left(K_{p}=100\right.$, dotted lines $)$ and presence of $K_{p}\left(K_{p}=0.5\right.$, bold lines) temperature profile increases as $R$ increases. It is also observed that for higher value of radiation parameter the temperature profile becomes linear. 


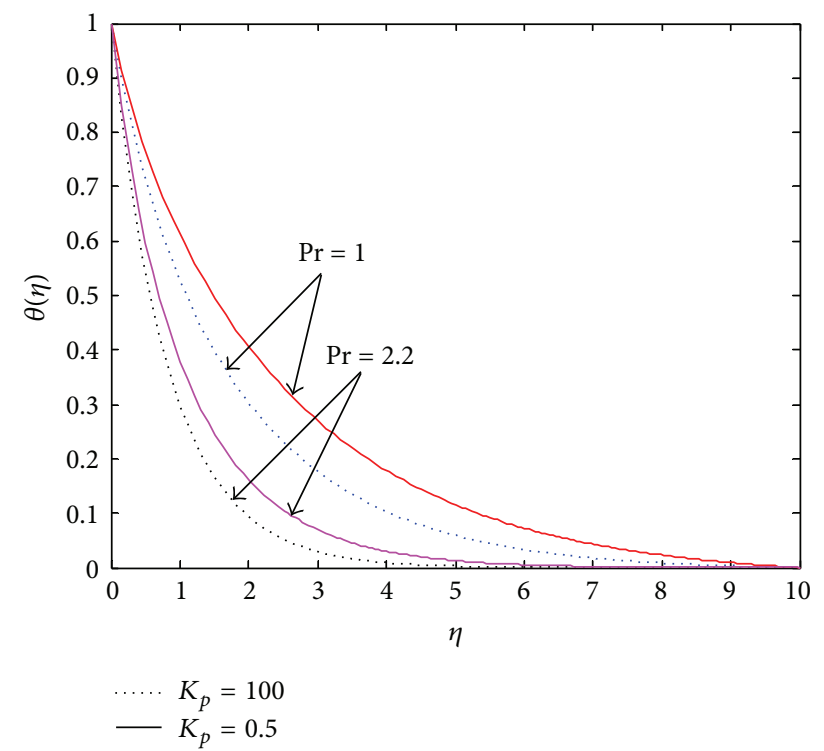

FIgURE 4: Temperature distribution for different values of $\operatorname{Pr}$ and $K_{p}$.

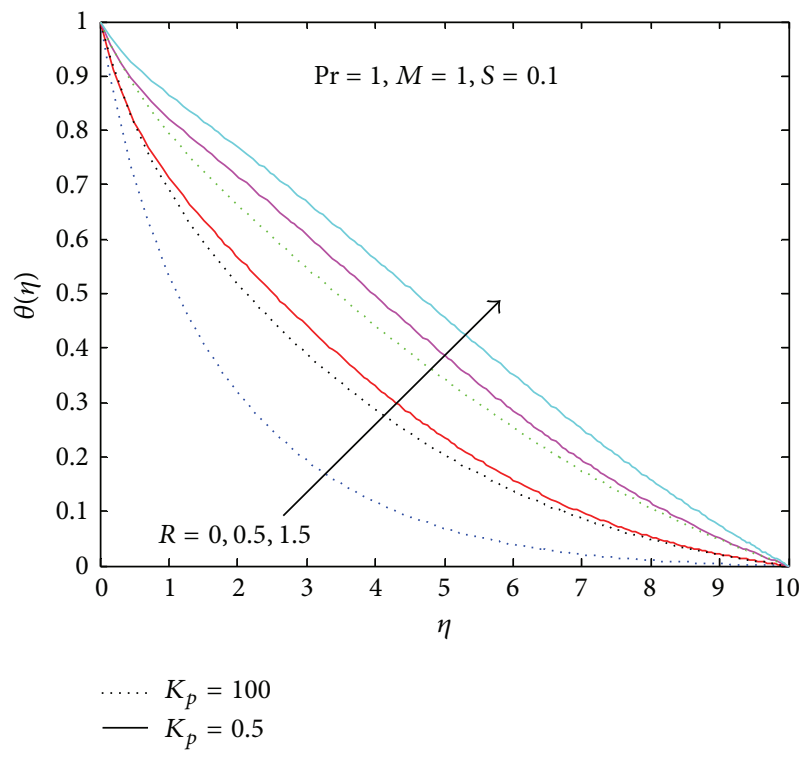

FIgURE 5: Temperature distribution for $R$ and $K_{p}$.

Figure 6 exhibits the effect of heat source/sink in both the absence and presence of the porous matrix. The striking feature of the temperature profiles is that an increase in $S$, that is, from sink to source, means a bigger amount of heat energy is stored due to thermal radiation leading to increase of the temperature at all points.

Figures 7 and 8 exhibit the concentration profiles for various values of the parameters characterizing the concentration distribution irrespective of the presence or absence of porous matrix. Now, from Figure 7 it is seen that the effect of magnetic field is to increase the concentration profile in both the presence and absence of porous matrix. Moreover, From Figure 8 it is noteworthy that an increase in Sc leads to decrease in concentration in both the absence and presence

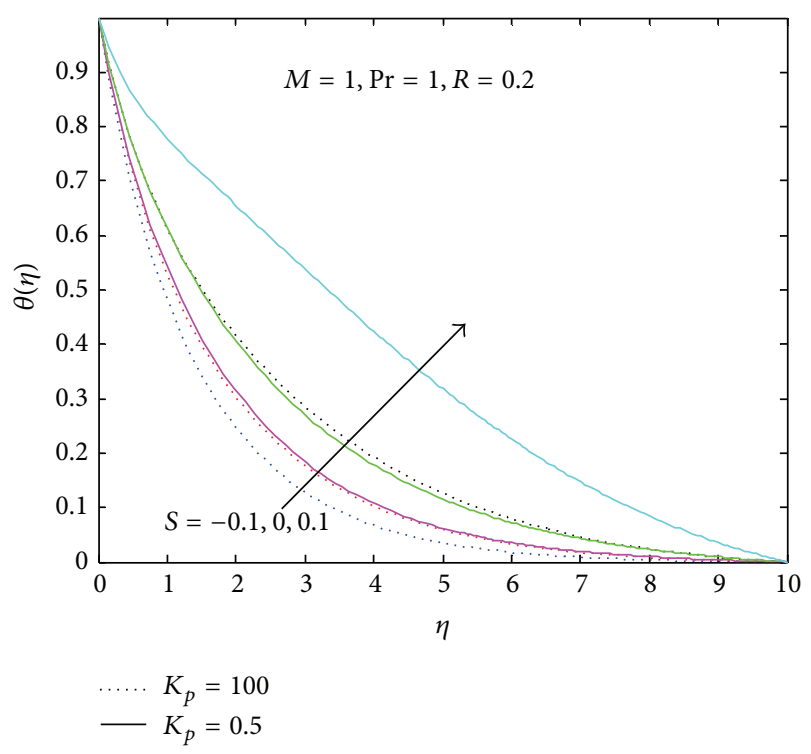

FIgURE 6: Temperature distribution for $S$ and $K_{p}$.

TABLE 1: Skin friction coefficient.

\begin{tabular}{lccc}
\hline Sl. number & $M$ & $K_{p}$ & $f^{\prime \prime}(0)$ \\
\hline 1 & 0 & 100 & 1.070259 \\
2 & 1 & 100 & 2.698484 \\
3 & 1 & 0.5 & 3.015113 \\
4 & 1 & 100 & 2.310844 \\
\hline
\end{tabular}

of porous matrix. Thus, heavier species contributes to retard the level of concentration in the presence/absence of porous matrix.

The effect of magnetic parameter and porosity parameter on skin friction coefficient is reflected in Table 1. From Table 1 it is observed that the increase in magnetic field parameter enhanced the skin friction coefficient as magnetic field creates Lorentz force which increases the value of skin friction coefficient. But the reverse effect is seen in case of porosity parameter. As $K_{p}$ increases, the skin friction coefficient decreases.

Similarly the effects of Prandtl number, radiation parameter, and source parameter on local Nusselt number are shown in Table 2. The local Nusselt number increases with increase in Prandtl number for $K_{p}=100$ and $K_{p}=0.5$. $N_{u}$ increases slowly as radiation parameter increases when $K_{p}=100$, but when $K_{p}=0.5$ it is seen that $N_{u}$ decreases as radiation parameter increases. Again at $K_{p}=0.5$ the local Nusselt number increases as source parameter increases due to storage of bigger amount of heat energy.

Table 3 shows the values of Sherwood number according to the variation of parameter $M$ and Sc. It is observed from the table that the Sherwood number decreases as magnetic parameter increases but increases when Schmidt number increases. 


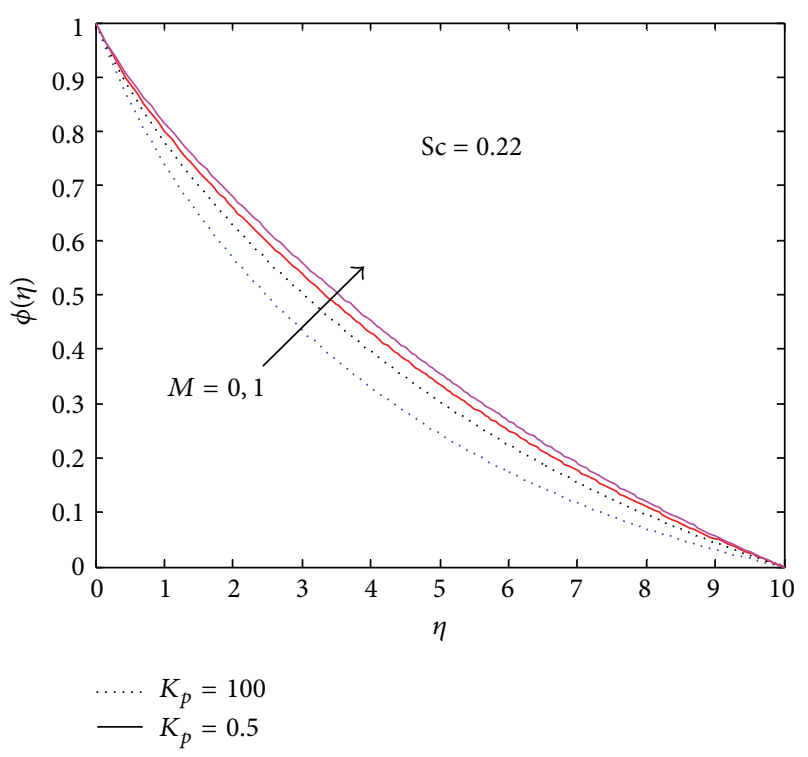

FIGURE 7: Concentration distribution for $M$ and $K_{p}$.

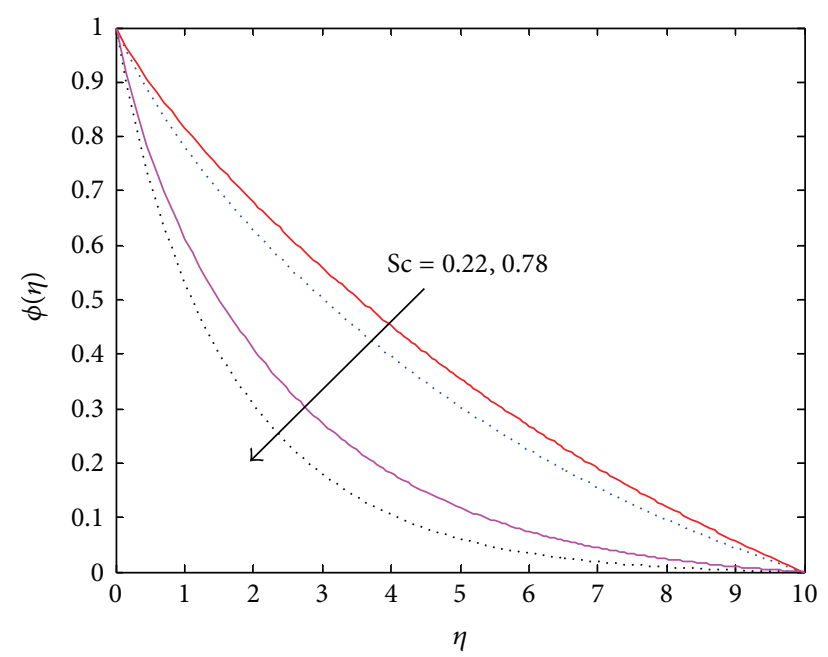

FIGURE 8: Concentration distribution for Sc and $K_{p}$.

TABLE 2: Values of Nusselt number $-\theta^{\prime}(0)$.

\begin{tabular}{cccccccc}
\hline$M$ & $K_{p}$ & $\operatorname{Pr}$ & $R$ & $S$ & Ishak et al. [25] & HAM & Present \\
\hline 0 & 100 & 1 & 1 & 0 & 0.5312 & 0.53121 & 0.53119 \\
0 & 100 & 2 & 1 & 0 & 1.4175 & 1.41751 & 1.41751 \\
0 & 100 & 1 & 0 & 0 & 0.9548 & 0.95478 & 0.9547 \\
0 & 0.5 & 1 & 1 & 0 & & & 0.4318 \\
0 & 0.5 & 2 & 1 & 0 & & & 1.2137 \\
0 & 0.5 & 1 & 0 & 0 & & & 0.7643 \\
1 & 100 & 1 & 1 & 0.1 & & & 0.9633 \\
1 & 0.5 & 1 & 1 & 0.1 & & & 0.7835 \\
\hline
\end{tabular}

\section{Conclusion}

In this paper we have investigated the effect of different flow parameters on dimensionless velocity, temperature,
TABLE 3: Values of Sherwood number $-\phi^{\prime}(0)$.

\begin{tabular}{lccc}
\hline Sl. number & $M$ & Sc & $-\phi^{\prime}(0)$ \\
\hline 1 & 3 & 0.6 & 1.331674 \\
2 & 3 & 0.22 & -1.90499 \\
3 & 3 & 0.1 & -2.35153 \\
4 & 4 & 0.6 & -2.14054 \\
5 & 4 & 0.78 & -1.90499 \\
\hline
\end{tabular}

and concentration profiles using Runge-Kutta method with shooting technique.

It is observed that

(i) magnetic field is decelerating the velocity profile and enhanced the skin friction coefficient due to resistive Lorentz force;

(ii) the temperature profile decreases for increasing value of Pr in the absence of porous matrix but obtained maximum value in the presence of porous matrix;

(iii) when radiation parameter increases the temperature profile increases; for higher value of radiation parameter the temperature profile becomes linear;

(iv) the concentration profile decreases with an increasing value of $S_{c}$ in both the absence and presence of porous matrix; thus heavier species contributes to retard the level of concentration in the presence/absence of porous matrix.

It is noticed that the results obtained will not only provide useful information for applications, but also give better accuracy comparing the result to Ishak et al. [25] and HAM.

\section{Conflict of Interests}

The authors declare that there is no conflict of interests regarding the publication of this paper.

\section{References}

[1] B. C. Sakiadis, "Boundary layer behaviour on continuous solid surface. Boundary layer equations for 2-dimensional and axisymmetric flow," AIChE Journal, vol. 7, pp. 26-28, 1961.

[2] B. C. Sakiadis, "Boundary-layer behavior on continuous solid surfaces: II. The boundary layer on a continuous flat surface," AIChE Journal, vol. 7, no. 2, pp. 221-225, 1961.

[3] T. Fang, "Similarity solutions for a moving-flat plate thermal boundary layer," Acta Mechanica, vol. 163, no. 3-4, pp. 161-172, 2003.

[4] T. Fang and C.-F. F. Lee, "A moving-wall boundary layer flow of a slightly rarefied gas free stream over a moving flat plate," Applied Mathematics Letters, vol. 18, no. 5, pp. 487-495, 2005.

[5] F. White, Viscous Fluid Flow, McGraw-Hill, New York, NY, USA, 3rd edition, 2006.

[6] B. C. Chandrasekhara, N. Radha, and M. Kumari, "The effect of surface mass transfer on buoyancy induced flow in a variable porosity medium adjacent to a vertical heated plate," Heat and Mass Transfer, vol. 27, no. 3, pp. 157-166, 1992. 
[7] A. J. Chamkha and A. Abdul-Rahim Khaled, "Hydromagnetic combined heat and mass transfer by natural convection from a permeable surface embedded in a fluid-saturated porous medium," International Journal of Numerical Methods for Heat and Fluid Flow, vol. 10, no. 5, pp. 455-476, 2000.

[8] O. D. Makinde, "Free convection flow with thermal radiation and mass transfer past a moving vertical porous plate," International Communications in Heat and Mass Transfer, vol. 32, no. 10, pp. 1411-1419, 2005

[9] O. D. Makinde, J. M. Mango, and D. M. Theuri, "Unsteady free convection with suction on an accelerating porous plate," Modelling, Measurement and Control, vol. 72, no. 3-4, pp. 3946, 2003.

[10] S. J. Kim and K. Vafai, "Analysis of natural convection about a vertical plate embedded in a porous medium," International Journal of Heat and Mass Transfer, vol. 32, no. 4, pp. 665-677, 1989.

[11] S.-J. Liao and I. Pop, "Explicit analytic solution for similarity boundary layer equations," International Journal of Heat and Mass Transfer, vol. 47, no. 1, pp. 75-85, 2004.

[12] S. U. S. Choi, "Enhancing thermal conductivity of fluids with nanoparticles," in Proceedings of the ASME International Mechanical Engineering Congress and Exposition, vol. 231/MD, no. 66, pp. 99-105, ASME Fluids Engineering Division, San Francisco, Calif, USA, November 1995.

[13] J. Buongiorno, "Convective transport in nanofluids," Journal of Heat Transfer, vol. 128, no. 3, pp. 240-250, 2006.

[14] S. Baag and S. R. Mishra, "Heat and mass transfer analysis on MHD 3-D water-based nanofluid," Journal of Nanofluids, vol. 4, no. 3, pp. 352-361, 2015.

[15] K. Vajravelu and D. Rollins, "Heat transfer in an electrically conducting fluid over a stretching surface," International Journal of Non-Linear Mechanics, vol. 27, no. 2, pp. 265-277, 1992.

[16] R. S. Tripathy, G. C. Dash, S. R. Mishra, and S. Baag, "Chemical reaction effect on MHD free convective surface over a moving vertical plane through porous medium," Alexandria Engineering Journal, vol. 52, no. 3, pp. 671-679, 2015.

[17] S. R. Mishra, G. C. Dash, and P. K. Pattnaik, "Flow of heat and mass transfer on MHD free convection in a micropolar fluid with heat source," Alexandria Engineering Journal, vol. 52, no. 3, pp. 681-689, 2015.

[18] E. M. Sparrow and J. P. Abraham, "A new buoyancy model replacing the standard pseudo-density difference for internal natural convection in gases," International Journal of Heat and Mass Transfer, vol. 46, no. 19, pp. 3583-3591, 2003.

[19] E. M. Sparrow and J. P. Abraham, "Universal solutions for the streamwise variation of the temperature of a moving sheet in the presence of a moving fluid," International Journal of Heat and Mass Transfer, vol. 48, no. 15, pp. 3047-3056, 2005.

[20] S. A. Al-Sanea, "Mixed convection heat transfer along a continuously moving heated vertical plate with suction or injection," International Journal of Heat and Mass Transfer, vol. 47, no. 6-7, pp. 1445-1465, 2004.

[21] V. M. Soundalgekar and T. V. Ramana Murty, "Heat transfer in flow past a continuous moving plate with variable temperature," Wärme- und Stoffübertragung, vol. 14, no. 2, pp. 91-93, 1980.

[22] R. Cortell, "Flow and heat transfer in a moving fluid over a moving flat surface," Theoretical and Computational Fluid Dynamics, vol. 21, no. 6, pp. 435-446, 2007.

[23] N. Afzal, A. Badaruddin, and A. A. Elgarvi, "Momentum and heat transport on a continuous flat surface moving in a parallel stream," International Journal of Heat and Mass Transfer, vol. 36, no. 13, pp. 3399-3403, 1993.

[24] A. Ishak, R. Nazar, and I. Pop, "The effects of transpiration on the flow and heat transfer over a moving permeable surface in a parallel stream," Chemical Engineering Journal, vol. 148, no. 1, pp. 63-67, 2009.

[25] A. Ishak, R. Nazar, and I. Pop, "Flow and heat transfer characteristics on a moving flat plate in a parallel stream with constant surface heat flux," Heat and Mass Transfer, vol. 45, no. 5, pp. $563-$ $567,2009$.

[26] P. M. Patil, S. Roy, and I. Pop, "Flow and heat transfer over a moving vertical plate in a parallel free stream: role of internal heat generation or absorption," Chemical Engineering Communications, vol. 199, no. 5, pp. 658-672, 2012.

[27] F. Mabood, W. A. Khan, and A. I. Md. Ismail, "MHD flow over exponential radiating stretching sheet using homotopy analysis method," Journal of King Saud University: Engineering Sciences, 2014. 

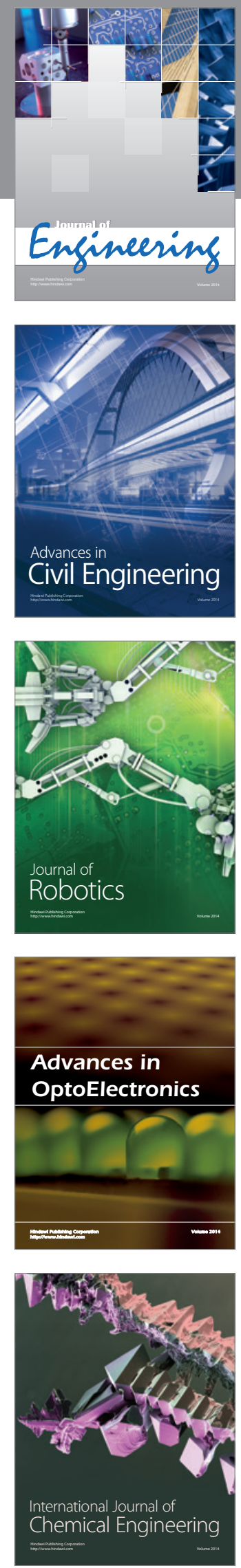

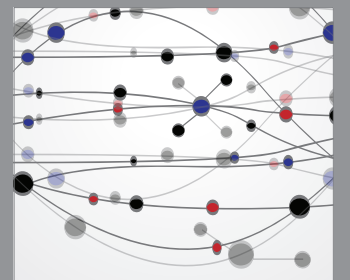

The Scientific World Journal
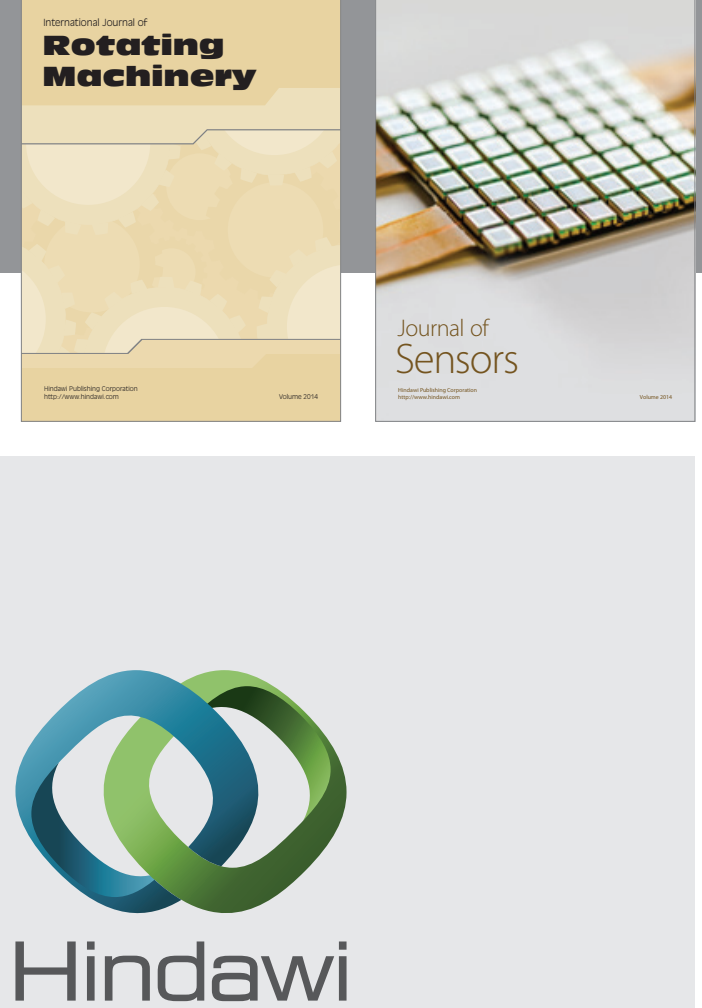

Submit your manuscripts at http://www.hindawi.com
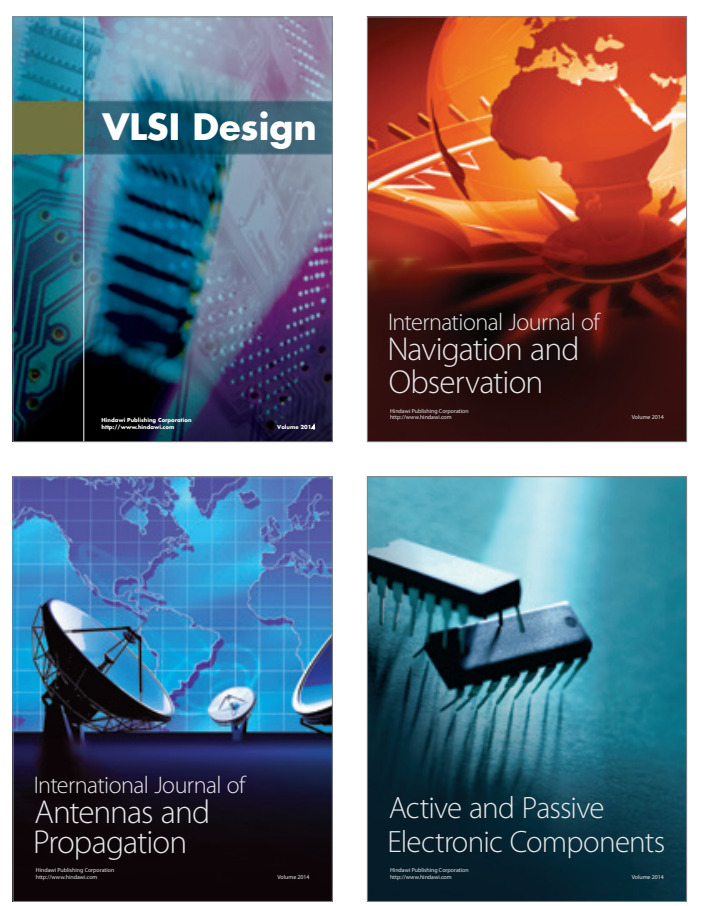
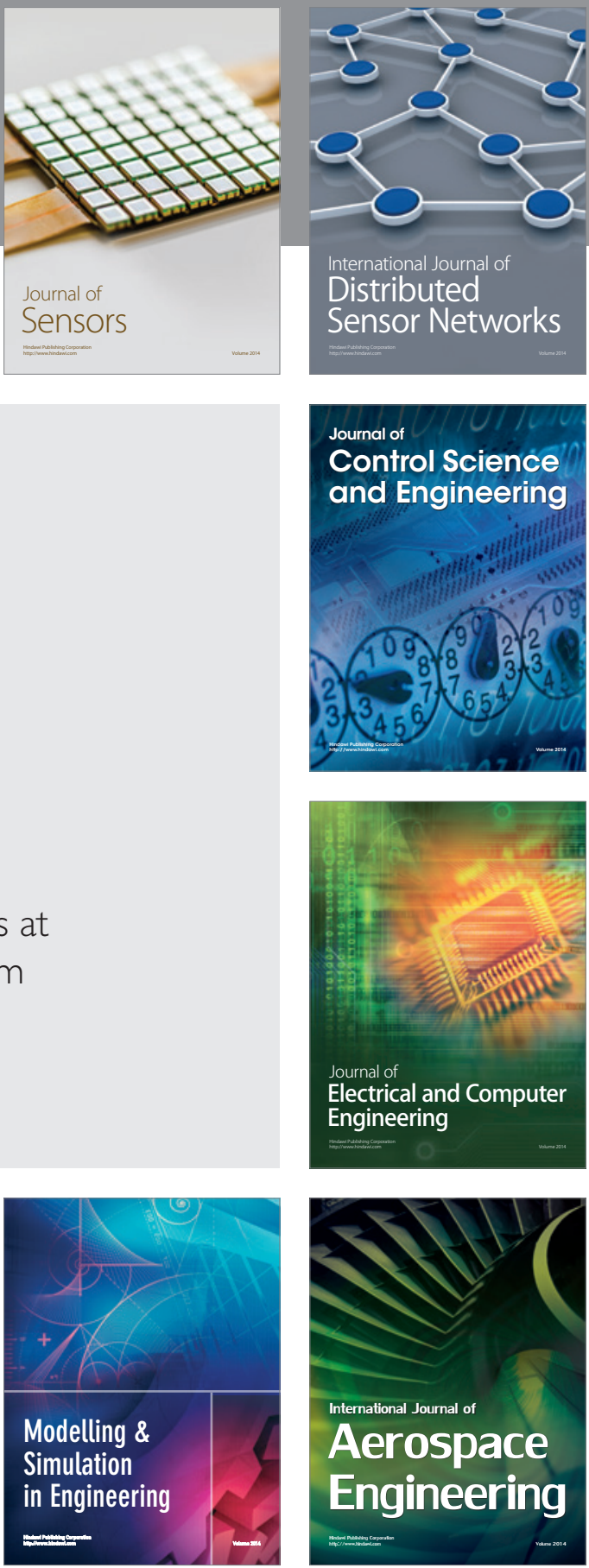

Journal of

Control Science

and Engineering
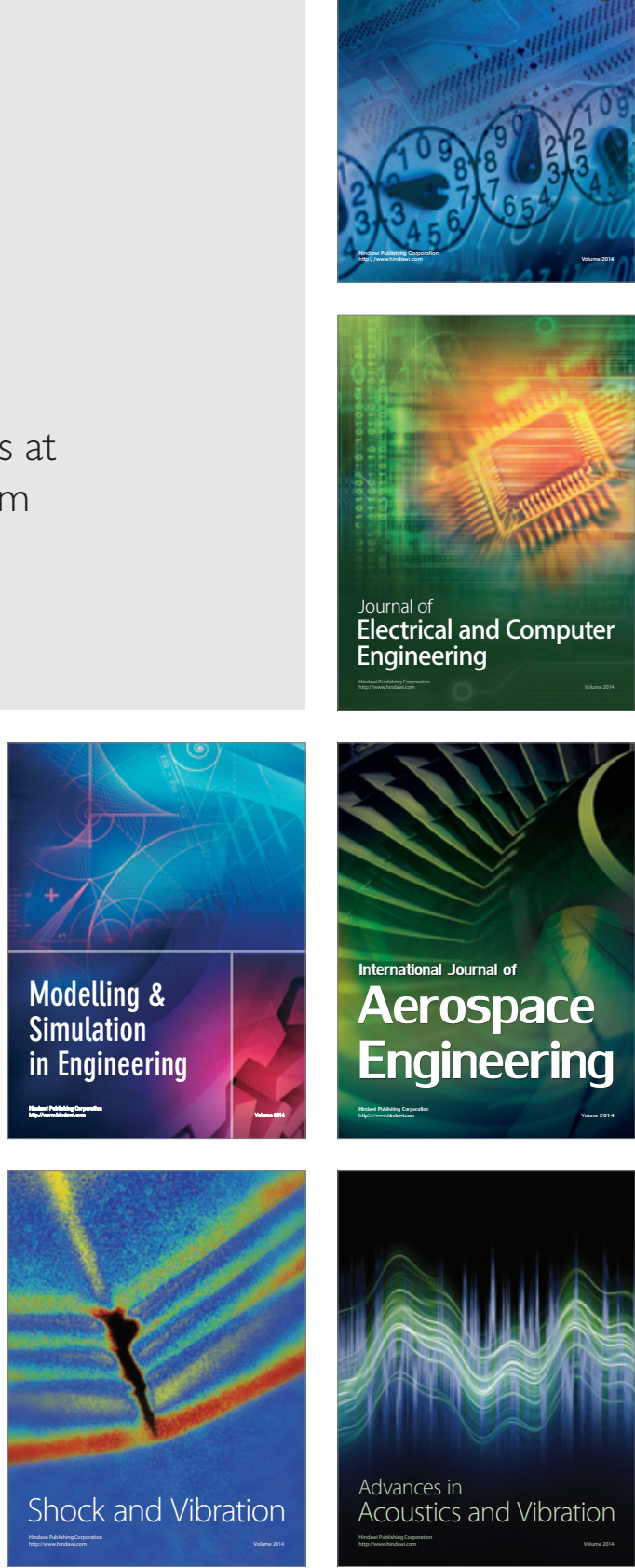